\title{
Dynamics of strongly coupled disordered dissipative spin-boson systems
}

\author{
Eliana Fiorelli, ${ }^{1,2}$ Pietro Rotondo, ${ }^{1,2}$ Federico Carollo, ${ }^{1,2,3}$ Matteo Marcuzzi, ${ }^{1,2}$ and Igor Lesanovsky ${ }^{1,2,3}$ \\ ${ }^{1}$ School of Physics and Astronomy, University of Nottingham, Nottingham, NG7 2RD, United Kingdom \\ ${ }^{2}$ Centre for the Mathematics and Theoretical Physics of Quantum Non-equilibrium Systems, University of Nottingham, \\ Nottingham NG7 2RD, United Kingdom \\ ${ }^{3}$ Institut für Theoretische Physik, Universität Tübingen, Auf der Morgenstelle 14, 72076 Tübingen, Germany
}

(Received 13 May 2019; accepted 20 November 2019; published 24 February 2020)

\begin{abstract}
Spin-boson Hamiltonians are an effective description for numerous quantum few- and many-body systems, such as atoms coupled to cavity modes, quantum electrodynamics in circuits, and trapped ion systems. While reaching the limit of strong coupling is possible in current experiments, the understanding of the physics in this parameter regime remains a challenge, especially when disorder and dissipation are taken into account. Here we investigate a regime where the spin dynamics can be related to an Ising energy function defined in terms of the spin-boson couplings. While in the coherent weak coupling regime it is known that an effective description in terms of spin Hamiltonian is possible, we show that a similar viewpoint can be adopted in the presence of dissipation and strong couplings. The resulting dynamics features approximately thermal regimes, separated by out-of-equilibrium ones in which detailed balance is broken. Moreover, we show that under appropriately chosen conditions one can even achieve cooling of the spin degrees of freedom. This points toward the possibility of using strongly coupled dissipative spin-boson systems for engineering complex energy landscapes together with an appropriate cooling dynamics.
\end{abstract}

DOI: 10.1103/PhysRevResearch.2.013198

\section{INTRODUCTION}

Prominent platforms for quantum simulation, such as cavity, circuit [1], or waveguide quantum electrodynamics [2] as well as trapped ions $[3,4]$ can be modeled by ensembles of two-level systems interacting via bosonic degrees of freedom (electromagnetic modes or phonons). While the weak coupling regime is relatively well understood and can be treated by a perturbative integration of the bosonic degrees of freedom, the strong coupling limit is far more challenging [5].

An additional layer of complexity is added by the presence of disorder, i.e., when individual spins couple to the bosonic "environment" at different strengths. Such a setting is relevant for at least two reasons. First, some degree of quenched disorder may always be present in realistic systems and, second, one may engineer nonuniform couplings for practical applications: Systems with tunable quasirandom couplings often form the basis for a physical implementation of complex optimization problems, which may for instance be solved via quantum annealing protocols $[6,7]$.

Disordered spin-boson systems have only recently moved into the focus of theoretical investigations. References [8,9] explore the emergence of glassiness when many electromagnetic modes interact with an ensemble of qubits. In Refs. [10,11], instead, spin-glass techniques are employed to show that the same system effectively realizes an associative

Published by the American Physical Society under the terms of the Creative Commons Attribution 4.0 International license. Further distribution of this work must maintain attribution to the author(s) and the published article's title, journal citation, and DOI. memory. Most of these techniques, however, cannot be straightforwardly generalized to study open quantum dynamics in the strong coupling regime, and only a few studies deal with disordered open quantum systems [12-15]. This topic acquires further relevance in the light of recent experimental progress in multimodal cavity QED, which realize tunable range [16] and sign-changing [17] photon-mediated atomic interactions.

In this work we investigate a disordered and dissipative system in which weakly driven spins are strongly coupled to a bosonic mode [see Fig. 1(a)]. We employ a perturbative approach which relies on the weakness of the driving rather than of the spin-boson coupling. We find that the effective spin dynamics is governed by a rate equation that depends on a fully connected Ising energy function as sketched in Fig. 1(b). Depending on the rates of bosonic loss and gain we identify several distinct dynamical regimes: Two of them are high-temperature ones, in which the stationary state of the system is fully mixed. A further one mimics an effective low-temperature dynamics, which permits cooling of the spin system. Outside these the dynamics is generally nonthermal and detailed balance is broken. This link between an open, strongly coupled spin-boson system and the physics of disordered Ising spin systems opens up the possibility of engineering complex classical energy landscapes-with importance in the context of optimization problems [18] or associative memories [19] - together with a cooling protocol.

\section{MODEL}

We consider an ensemble of $N$ two-level systems interacting with a single bosonic mode described by the following 


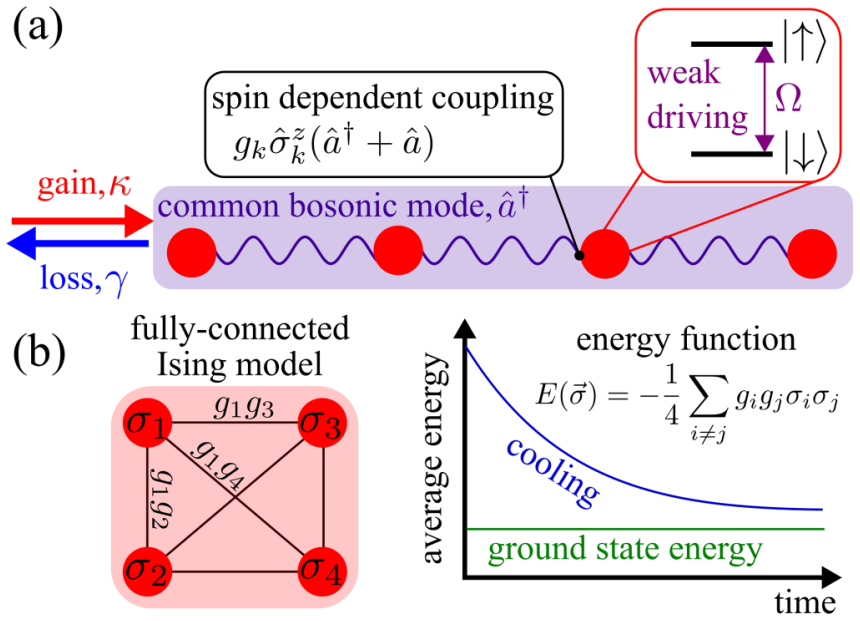

FIG. 1. Dissipative spin-boson system. (a) $N$ weakly driven (at strength $\Omega$ ) two-level systems (spins) are strongly coupled to a single bosonic mode with couplings $g_{k}(k=1, \ldots, N)$. Gain and loss of the bosons occur at rates $\kappa$ and $\gamma$, respectively. (b) The resulting effective dissipative dynamics of the spins is related to a fullyconnected Ising energy function $\left(\sigma_{k}= \pm 1\right), E(\vec{\sigma})$ [Eq. (5)], in which the interaction strength between spins $i$ and $j$ is proportional to $g_{i} g_{j}$. The effective dynamics features regimes which permit cooling of the collective spin state, i.e., significant population of the low-energy configurations.

Dicke Hamiltonian [20-23]:

$$
\hat{H}=\omega \hat{a}^{\dagger} \hat{a}+\sum_{i=1}^{N} g_{i} \hat{\sigma}_{i}^{z}\left(\hat{a}^{\dagger}+\hat{a}\right)+\Omega \sum_{i=1}^{N} \hat{\sigma}_{i}^{x} .
$$

Here, $\hat{\sigma}_{i}^{x, y, z}$ are the Pauli operators and $\hat{a}$ and $\hat{a}^{\dagger}$ the bosonic annihilation and creation operators. The parameters $\omega$ and $\Omega$ denote the fundamental frequency of the bosons and the coherent coupling strength between the two spin states, respectively. The spin-boson couplings $g_{i}$ will be modeled here as a set of independent, identically distributed real random variables. In the following we shall consider two cases: As a representative of distributions centered around zero, we first take a uniform one with weight $1 /\left(2 g_{0}\right)$ between $-g_{0}$ and $g_{0}$ and zero elsewhere. We then consider a bimodal distribution which peaks around two opposite values $\pm g_{0}$. This choice mimics the two-value distribution often encountered in associative memory models $[10,11,19]$. More precisely, we superpose two Gaussians, each of variance $s^{2}$, centered around $\pm g_{0}$. In both cases the mean is zero and we denote the variance by $g^{2}$.

We also include dissipation on the bosonic degree of freedom in the form of Markovian gain and loss processes. The state of the system, encoded in a density matrix $\rho$, thus evolves under the action of a Lindblad equation

$$
\dot{\rho}=\mathcal{L} \rho=-i[\hat{H}, \rho]+\sum_{n=l, g} \hat{L}_{n} \rho \hat{L}_{n}^{\dagger}-\frac{1}{2}\left\{\hat{L}_{n}^{\dagger} \hat{L}_{n}, \rho\right\},
$$

with the jump operators $\hat{L}_{l}=\sqrt{\gamma} \hat{a}, \hat{L}_{g}=\sqrt{\kappa} \hat{a}^{\dagger}$, where $\gamma(\kappa)$ is the loss (gain) rate and $\gamma>\kappa \geqslant 0$.

Let us at this point briefly discuss the validity of the employed Lindblad master equation description. This is a formalism widely employed for dissipative light-matter systems [21,24-26]. Formally, it is derived from a setting in which work is performed upon the environment so that its correlation with the system is very quickly erased (on timescales smaller than the ones at which correlations are built between different subsystems, in this case spins and bosons). Equivalently, one can imagine that the system is coupled in succession to a plethora of equal copies of the environment [27]. Constant contact with a single environment would eventually correlate system and bath degrees of freedom; in the system dynamics, this would be reflected in the emergence of nonlocal terms. For instance, in our description this would produce dissipation on the spins as well, neglecting which would cause apparent violations of the second law of thermodynamics [28].

A physical realization of the Dicke model can, for instance, be achieved on trapped-ion quantum simulators, as it has been addressed both theoretically $[29,30]$ and experimentally $[31,32]$. Following the scheme represented in Fig. 1, such a system would consist of $N$ ions coupling to the center-of-mass phonon mode of the lattice. As it has been shown for the quantum Rabi model [33] and eventually generalized to the Dicke model [30], the application of multiple laser fields on the ions yields both the spin dependent coupling $g_{k} \hat{\sigma}_{k}^{z}\left(\hat{a}+\hat{a}^{\dagger}\right)$ and the weak driving term, $\Omega \hat{\sigma}_{k}^{x}$ entering Eq. (1). Finally, as illustrated in Fig. 1(a), the gain and loss dynamics can be achieved by applying lasers on the ions on the edge of the chain, as discussed in Ref. [34]. Since this ion is coupled to the same phonon mode as the other ions this effectively implements jump operators of the form introduced in Eq. (2).

\section{SPIN DYNAMICS AT STRONG COUPLING}

We explore the dynamics Eq. (2) in the strong coupling regime, i.e., when the driving acting on the spins is much weaker than the spin-boson interaction $(\Omega \ll g)$. In the following, we sketch the perturbative technique we employ for this purpose. First, we split the Lindblad superoperator according to $\mathcal{L}=\mathcal{L}_{0}+\mathcal{L}_{1}$, where $\mathcal{L}_{1}(\cdot)=-i \Omega\left[\sum_{i} \hat{\sigma}_{i}^{x}, \cdot\right]$ can be regarded as a small perturbation. Focusing now on $\mathcal{L}_{0}$, we notice that each $\hat{\sigma}_{i}^{z}$ commutes with all jump operators and Hamiltonian terms in it, implying that the $z$ components of the spins constitute $N$ independent conserved quantities [35]. Hence, the dynamics can be separated in $2^{N}$ independent sectors labeled by the classical spin configurations $\vec{\sigma}=$ $\left(\sigma_{1}, \ldots, \sigma_{N}\right)\left(\sigma_{i} \in\{-1,1\}\right)$, where $\hat{\sigma}_{i}^{z}|\vec{\sigma}\rangle=\sigma_{i}|\vec{\sigma}\rangle$; in other words, states belonging to different sectors never mix under the action of $\mathcal{L}_{0}$. In each sector, the bosonic mode evolves according to a reduced Lindbladian $\mathcal{L}_{0}\left(\hat{\sigma}_{i}^{z} \rightarrow \sigma_{i}\right)$ in which each Pauli matrix is replaced with the corresponding eigenvalue extracted from the chosen label. For any such choice of classical configuration, the reduced Lindbladian is quadratic in the bosonic operators and thus describes a damped quantum harmonic oscillator with a (spin-configuration-dependent) spatial displacement. This admits a single (bosonic) stationary state, denoted by $\rho_{\vec{\sigma}}$. We assume that, due to the random and independent nature of the couplings $g_{i}$, no additional symmetries are present which could protect more complex subspaces. Hence, for any initial state $\rho_{0}$ of the spin-boson system the corresponding stationary state under $\mathcal{L}_{0}$ is of the 
form $\rho_{\text {stat }}=\sum_{\vec{\sigma}} p_{\vec{\sigma}} \rho_{\vec{\sigma}} \otimes|\vec{\sigma}\rangle\langle\vec{\sigma}|$, where the coefficients $p_{\vec{\sigma}}$ form a set of classical probabilities.

The perturbation $\mathcal{L}_{1}$ couples sectors corresponding to different classical spin configurations $\vec{\sigma}$. Its action can be incorporated perturbatively $[36,37]$ as long as $\Omega$ is small compared to the typical rate at which coherences between sectors decay (estimated further below). We define the projector $P$ onto the stationary manifold of $\mathcal{L}_{0}$ according to

$$
P \rho(t)=\sum_{\vec{\sigma}} \operatorname{Tr}_{B}\{\langle\vec{\sigma}|\rho(t)| \vec{\sigma}\rangle\} \rho_{\vec{\sigma}} \otimes|\vec{\sigma}\rangle\langle\vec{\sigma}|,
$$

where $\operatorname{Tr}_{B}$ denotes the partial trace over the bosonic mode, and use it to define a projected dynamics for $P \rho(t)$. This is a particular example of a Nakajima-Zwanzig projection scheme [38]. In our case, this reduction yields a considerable simplification: From Eq. (3) we see in fact that the time dependence of the reduced state is entirely encoded in the coefficients $p_{\vec{\sigma}}(t)=\operatorname{Tr}_{B}\{\langle\vec{\sigma}|\rho(t)| \vec{\sigma}\rangle\}$, which constitute a list of classical probabilities. These evolve according to a master equation $\dot{p}_{\vec{\sigma}}=\sum_{\vec{\sigma}^{\prime}} W_{\vec{\sigma}^{\prime} \rightarrow \vec{\sigma}} p_{\vec{\sigma}^{\prime}}-W_{\vec{\sigma} \rightarrow \vec{\sigma}^{\prime}} p_{\vec{\sigma}}$, where $W_{\vec{\sigma}^{\prime} \rightarrow \vec{\sigma}}$ is the rate for switching from configuration $\vec{\sigma}^{\prime}$ to $\vec{\sigma}$. Note that, up to second order in $\Omega$, the corresponding stochastic process includes only single spin flips (i.e., $W_{\vec{\sigma}^{\prime} \rightarrow \vec{\sigma}} \neq 0$ only if $\vec{\sigma}$ and $\vec{\sigma}^{\prime}$ differ by a single spin). The rates are derived in Appendix A; here we limit ourselves to provide their final expression:

$$
\begin{aligned}
W_{\vec{\sigma} \rightarrow \vec{\sigma}^{\prime}} & =\frac{2 \Omega^{2}}{\omega} \int_{0}^{\infty} d \tau e^{-\frac{2 g_{i}^{2} \nu}{\omega^{2}}(f(\tau)+\tau)} \cos \left[16 \frac{\Delta E_{i} \tau-g_{i}^{2} s(\tau)}{\omega^{2}\left(\eta^{2}+4\right)}\right], \\
f(\tau) & =\frac{8-2 \eta^{2}}{\eta\left(\eta^{2}+4\right)}\left[1-e^{-\frac{\eta}{2} \tau} \cos (\tau)\right]-\frac{8 e^{-\frac{\eta}{2} \tau}}{\eta^{2}+4} \sin (\tau), \\
s(\tau) & =\frac{4 \eta\left[e^{-\frac{\eta}{2} \tau} \cos (\tau)-1\right]+\left[\eta^{2}-4\right] e^{-\frac{\eta}{2} \tau} \sin (\tau)}{\eta^{2}+4}
\end{aligned}
$$

where the index $i$ denotes which spin is being flipped and changes sign between configurations $\vec{\sigma}$ and $\vec{\sigma}^{\prime}$.

In Eq. (4) we have introduced for brevity the (scaled) difference between loss and gain rates $\eta=(\gamma-\kappa) / \omega \equiv$ $\gamma / \omega(1-\theta)$, the ratio $\theta=\kappa / \gamma \in[0,1)$ and the parameter $v=4(1+\theta) \eta /\left[\left(\eta^{2}+4\right)(1-\theta)\right]$. Importantly, the sole dependence on the spin configuration is through the quantity $\Delta E_{i}=g_{i} \sigma_{i} \sum_{l \neq i} g_{l} \sigma_{l}$, which can be interpreted as an energy difference (see further below). Note that there is a characteristic scale of exponential suppression of the integrand of $W_{\vec{\sigma} \rightarrow \vec{\sigma}^{\prime}}$. This corresponds to the typical timescale involved in the loss of coherence between sectors belonging to different classical spin configurations. Since the function $f(\tau)$ is bounded, and thereby $f(\tau)+\tau \sim \tau$ for $\tau \rightarrow \infty$, we can estimate this timescale as $t_{\mathrm{L}}=\tau_{L} / \omega \approx \omega /\left(2 g^{2} \nu\right)$. Our perturbative expansion thus holds as long as $\Omega \ll 1 / t_{\mathrm{L}}$.

In the following we perform a detailed investigation of the effective spin dynamics. It turns out that the loss-gain parameter $\eta$ is central in determining the qualitative dynamical behavior: We will identify an effective high-temperature regime in the asymptotic limits $\eta \rightarrow \infty$ and $\eta \rightarrow 0^{+}$. Furthermore, we find an effective low-temperature (cooling) dynamics when $\eta<1$ and $\theta$ is close to unity.

\section{LARGE $\eta$ : INFINITE TEMPERATURE DYNAMICS}

As remarked above, the quantity $\Delta E_{i}$ can be interpreted as the change in the energy function

$$
E(\vec{\sigma})=-\frac{1}{4} \sum_{i \neq j} g_{i} g_{j} \sigma_{i} \sigma_{j}
$$

occurring when the $i$ th spin is flipped, i.e., $\Delta E_{i}=E\left(-\sigma_{i}\right)-$ $E\left(\sigma_{i}\right)$. For a large gain-loss difference, $\eta \gg 1$, we find that in Eq. (4) $f(\tau) \sim s(\tau) \sim O(1 / \eta)$. Therefore, both functions are approximately zero and the parameter $v \approx 4 \frac{1+\theta}{1-\theta} \eta^{-1}$ determines the leading behavior of the timescale $t_{\mathrm{L}}$. The validity of the perturbative requirement thus imposes an upper bound to the loss-gain difference, which must satisfy $1 \ll \eta \ll$ $\frac{4 g^{2}(1+\theta)}{\omega \Omega(1-\theta)}$.

With the above approximations the rate $W_{\vec{\sigma} \rightarrow \vec{\sigma}^{\prime}}$ acquires a considerably simpler form: having neglected $s(\tau)$, it no longer depends on the sign of $\Delta E_{i}$, implying that the rates for inverse processes $\sigma \rightarrow \sigma^{\prime}$ and $\sigma^{\prime} \rightarrow \sigma$ are equal. This gives rise to an infinite-temperature dynamics which populates all configurations uniformly. This behavior is highlighted in Fig. 2(a) for an example using couplings $g_{i}$ extracted from a uniform distribution: We show that the average energy $\langle E\rangle(t)$ approaches (up to small corrections) zero, its infinite-temperature average, as a consequence of reaching an approximately equal population of all spin configurations at stationarity.

Interestingly, for large $\eta$ and up to second order in perturbation theory, the rate $W_{\vec{\sigma} \rightarrow \vec{\sigma}^{\prime}}$ is formally equivalent to the dissipative dynamics of a fictitious transverse field Ising model. The corresponding Hamiltonian is $\hat{H}_{\mathrm{eff}}=$ $\Omega_{\text {eff }} \sum_{i} \hat{\sigma}_{i}^{x}+\xi E\left(\hat{\sigma}^{z}\right)$ [with $\Omega_{\text {eff }}=\Omega \lambda, \xi=8 \lambda^{2} /\left(\omega \eta^{2}\right)$ ] and the spins are subject to strong dephasing at a (site-dependent) rate $\gamma_{\mathrm{eff}, \mathrm{i}}=\frac{8 g_{i}^{2} \lambda^{2}(1+\theta)}{\omega \eta(1-\theta)}$ [39]. Here $\lambda$ is an arbitrary factor that should be chosen consistently with the (perturbative) requirement $\Omega_{\text {eff }} / \gamma_{\text {eff,i }} \ll 1$. Therefore, in this limit, the bosons can be interpreted as forming an infinite temperature bath causing dephasing of the spin degrees of freedom.

\section{SMALL $\eta$ : APPROXIMATE LOW-TEMPERATURE DYNAMICS}

We now consider the opposite case $0<\eta \ll 1$; as we discuss further below, a regime can be found in which the rate equation dynamics mimics, to an extent, a thermal process with finite temperature. To start, we keep $\theta$ as an independent parameter and consider couplings extracted from the same uniform distribution employed above. As shown in Fig. 2(b), in this regime the stationary average of the energy function Eq. (5) generally approaches values distinctively lower than the infinite temperature one found in the previous section. Moreover, our numerical exploration of this parameter regime suggests that this "cooling" tendency is enhanced as $\theta$ approaches its upper limit 1 . An effect of this can be gleaned from the two curves for $\theta=0.5$ (dashed line) and 0.9 (solid line) displayed in Fig. 2(b), where the latter clearly decreases to a lower value than the former. From a physical standpoint, recalling that $\theta=\kappa / \gamma$ describes the relative strength of losses and gains, this suggests that the bosonic mode cools 
(a) large gain-loss difference

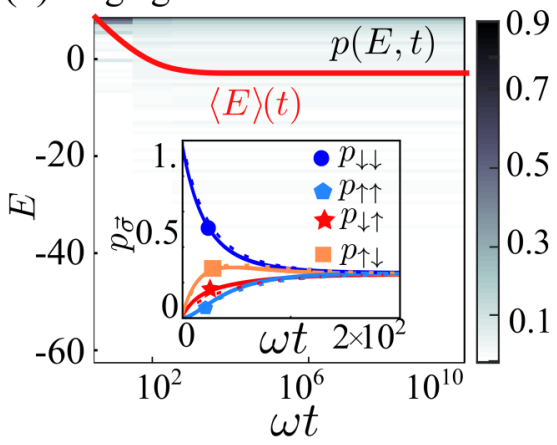

(b) small gain-loss difference

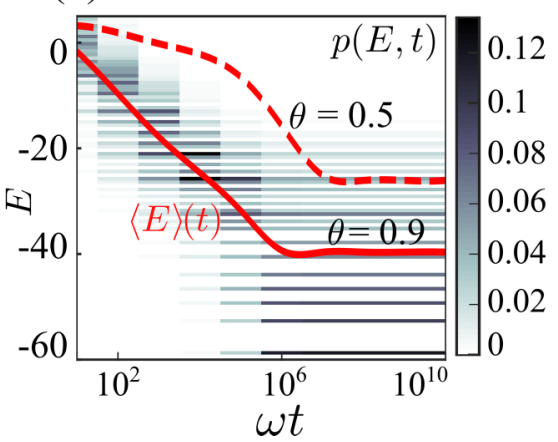

(c)

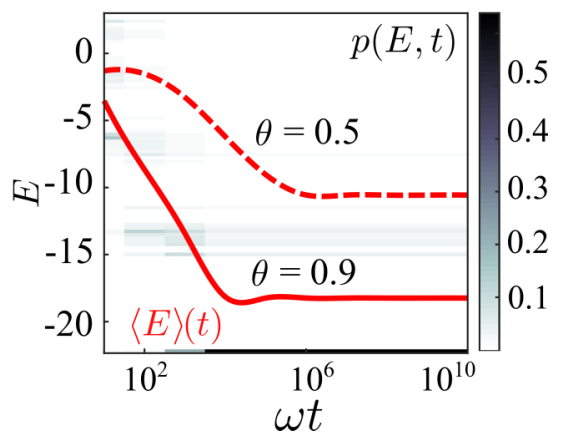

FIG. 2. Regimes of effectively thermal spin dynamics. We set $\Omega=0.1, \omega=1$. (a) Statistical energy distribution versus time for $N=10$ spins for large gain-loss difference $\eta$, starting from an initial state where all spins point down. The superposed red solid line displays the evolution of the average energy, $\langle E\rangle(t)$ as a function of time, whereas the shading represents the probability of being in a configuration with energy $E$ at time $t$. We set $\eta=10$ and $\theta=1 / 11$ and we select the couplings $g_{i}$ from a uniform distribution in $\left[-g_{0}, g_{0}\right]$ with $g_{0}=3$. Inset: evolution of the probabilities $p_{\sigma}$ for a system of two spins with the same parameters. We compare the effective model (solid lines) with the numerically exact diagonalization of the full open quantum problem (dashed lines), highlighting good agreement. (b) Statistical energy distribution versus time for $N=10$ spins and corresponding evolution of the average energy, $\langle E\rangle(t)$ for $\eta=0.1$ and $\theta=0.9$ for the red solid line and $\theta=0.5$ for the red dashed line. We select the couplings $g_{i}$ as in (a). The shading represents the probability density of the energy levels and refers to the latter case $(\theta=0.9)$. (c) The same analysis of (b) has been performed with $g_{i}$ having been selected from a bimodal distribution given by the symmetric superposition of two Gaussians centered in $\pm g_{0}= \pm 1$ and variance $s=0.25$. Again, the dynamics tends to preferentially populate the low-energy configurations at long times, and the more so the closer $\theta$ is to 1 . As for (b), the shading relates to the $\theta=0.9$ case.

the spins more effectively when higher bosonic numbers are present.

In the third panel of Fig. 2 we repeat the same analysis for a set of couplings $g_{i}$ produced from a different distribution. We take in this case a symmetric, bimodal one to mimic an associative memory model [19] with the following important differences: First, as pointed out in previous works [10,11], accounting for a single bosonic mode implies that only a single pattern (and its complementary, where all spins are flipped) can be stored in the system, rendering the memory almost trivial. Second, in previous works the couplings can only take two possible values $\pm g_{0}$ with equal probability. For consistency with our assumptions that no higher symmetries than the ones we identified at the beginning are present, in our work we cannot allow the couplings to be equal in pairs (otherwise the total spin on the pair of sites would be conserved). Hence, we broaden the peaks into two Gaussians, identical up to a translation, centered at $\pm g_{0}$ with variance $s^{2}$. In other words, if we denote by $\mathcal{N}_{g_{0}, s}(g)=\left(2 \pi s^{2}\right)^{-1 / 2} \exp \left[-\left(g-g_{0}\right)^{2} /\left(2 s^{2}\right)\right]$ a generic normal distribution, we generate our couplings from

$$
p(g)=\frac{1}{2}\left[\mathcal{N}_{g_{0}, s}(g)+\mathcal{N}_{-g_{0}, s}(g)\right] .
$$

With this choice, the cooling behavior is still present and displays the same qualitative features highlighted for the uniform case, i.e., the dynamics tends to explore lower energies for values of $\theta$ closer to 1 .

A naive comparison of Figs. 2(b) and 2(c) suggests that the bosons are more effective in their cooling action on the spins when the spin-boson couplings are bimodally distributed. In fact, one can notice a sharper contrast between the shading (proportional to the population) of the ground state with respect to the other energy levels in Fig. 2(c) than in Fig. 2(b). However, the two distributions have different variances and the scale of the shading is different in the two panels. To more properly address this difference, we have compared the bimodal distribution used in Fig. 2(c) with a uniform one with the same variance, i.e., with $g_{0}=\sqrt{51} / 4$. Figure 3 displays a counting diagram of the stationary ground-state population for 1000 realisations of either distribution of the couplings. Here, one can notice that the bimodal case has, statistically speaking, a distinctively higher ground-state population than the uniform case, peaking beyond 0.5. Uniformly distributed couplings, in contrast, give rise to dynamics which typically populate the ground state much less, with a finite probability of having less than $5 \%$ population at long times.

\section{THERMAL VERSUS NONTHERMAL RATES}

To obtain some insight on this cooling mechanism we extract now some approximate expressions for the transition rate, which, for simplicity, we treat as a function of the energy difference $\Delta E$, now regarded as a continuous parameter. Additionally, to simplify the treatment we restrict to the case where $\eta$ is made smaller by bringing $\gamma \rightarrow \kappa^{+}$, while keeping $\kappa$ and $\omega$ constant, so that $\theta=(1+\eta \omega / \kappa)^{-1}$ increases toward unity as $\eta$ is decreased. Despite being a restriction, this allows us to explore the most interesting regime, i.e., the one where the dynamics seems to achieve the most efficient cooling. The procedure is discussed in some detail in Appendix B, Below we briefly comment on the main steps involved. First, we note that, for sufficiently small $\eta$, the integrand defining $W_{\vec{\sigma} \rightarrow \vec{\sigma}^{\prime}}$ is rapidly suppressed for $\tau>0$ due to a fast initial increase of $f(\tau) \approx 2(1-\cos \tau) / \eta$. Thus, the integral is dominated by the contribution close to $\tau=0$. Hence, one can expand all arguments in powers of $\tau$, see Eqs. (B2) and (B3). Setting for simplicity $\omega=\kappa=1$ and keeping for brevity only the leading orders in $\eta \rightarrow 0$, one obtains $\tau+f(\tau) \approx \tau^{2} / \eta-\tau^{3} / 6, v \approx 2$ and $s(\tau) \approx-\tau+\tau^{3} / 6$. This implies that the suppression of 
$\square$ Bimodal Distribution $\square$ Uniform Distribution

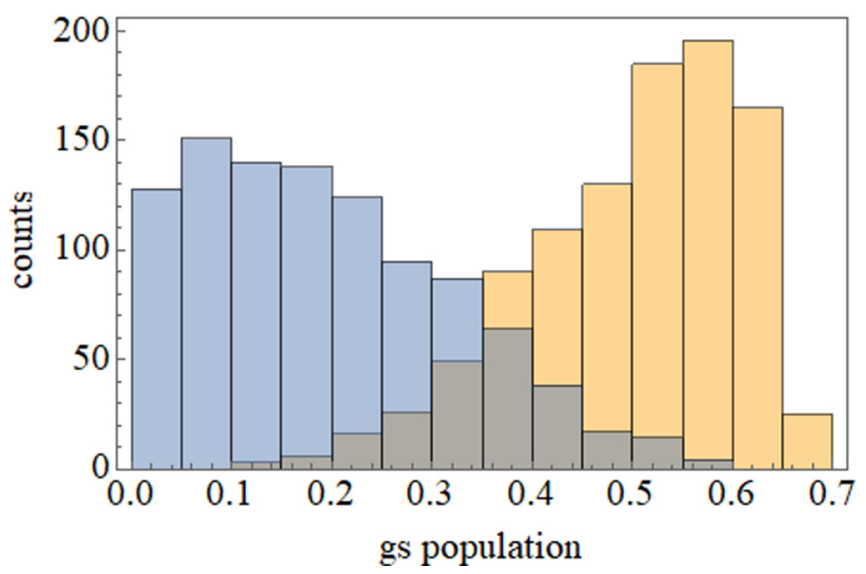

FIG. 3. Ground-state population for two different distributions of the spin-boson couplings. Histogram of the stationary groundstate (gs) population for 1000 realisations of either distributions. Blue (yellow) columns refer to the uniform (bimodal) case. Both distributions have zero mean and variance $g^{2}=17 / 16$. The physical parameters are $\omega=1, \eta=0.1, \theta=1 /(\eta+1) \approx 0.909, \Omega=1$ for a system of $N=10$ spins. The bimodal distribution data peaks beyond 0.5 ; the extracted mean is approximately $50 \%$ with a standard deviation of $11 \%$, implying half of the final population typically lies in the ground state; the tails show a negligible probability of it being smaller than $20 \%$. Conversely, the uniform distribution generates a slightly broader profile, with an average of approximately $20 \%$ and a standard deviation of $13 \%$, implying that the ground state is populated much less in proportion.

the integrand occurs on a timescale $\tau \sim \sqrt{\eta /\left(4 g_{i}^{2}\right)}$, whereas the cosine term oscillates with a frequency which is approximately $\Gamma_{i}=4\left(\Delta E+g_{i}^{2}\right)$. We thereby identify (i) a regime of "small energy jumps," where $\Gamma_{i}^{2} \ll 4 g_{i}^{2} / \eta$ and (ii) a "large energy jumps" one with $\Gamma_{i}^{2} \gg 4 g_{i}^{2} / \eta$. In case (i), we obtain

$$
W_{i}(\Delta E) \approx \frac{\Omega^{2}}{2} \sqrt{\frac{\pi \eta}{g_{i}^{2}}} e^{-\frac{\eta}{g_{i}^{2}}\left(\Delta E+g_{i}^{2}\right)^{2}},
$$

where the index $i$ reminds us of which spin is being flipped [40]. The rate reaches its maximum when $\Delta E=-g_{i}^{2} \leqslant 0$ and, in general, $W_{i}(|\Delta E|)<W_{i}(-|\Delta E|)$. This means that spin flips which lower the energy are favored, consistently with the picture of a cooling dynamics. Case (ii) can be analyzed using the asymptotic expansion of Fourier integrals [41], Eq. (B11). To leading order this yields a power-law decay $W_{i}(\Delta E) \approx$ $8 \Omega^{2} g_{i}^{2} \Gamma_{i}^{-4}$, which shares a similar preference for spin flips which lower the energy. A numerical integration suggests that $W_{i}(|\Delta E|)<W_{i}(-|\Delta E|)$ holds also in between the asymptotic cases (i) and (ii).

To shed further light on the cooling dynamics we analyze this asymmetry of the rates through the ratio $R_{i}(\Delta E)=$ $W_{i}(\Delta E) / W_{i}(-\Delta E)$. This is depicted in Figs. 4(a) and 4(b) as a function of $\Delta E$ and $\eta$, respectively. In regime (i) we have $R_{i}(\Delta E) \approx e^{-4 \eta \Delta E}$, which implies a thermal dynamics with an effective inverse temperature $\beta_{\text {eff }}=4 \eta$. Note that the righthand side has no dependence on the index $i$, implying the existence of a unique, well-defined temperature for all spin-flip processes. In Fig. 4(c) we display the ratio $\log \left[R_{i}(\Delta E)\right] / \Delta E$ (a)

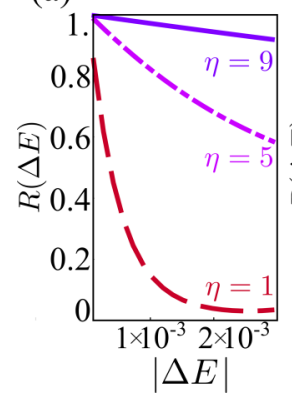

(b)

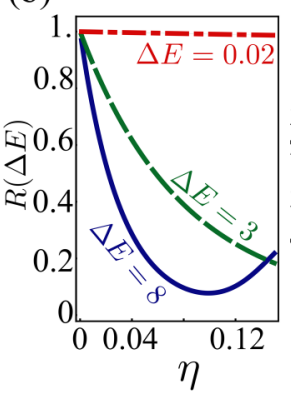

(c)

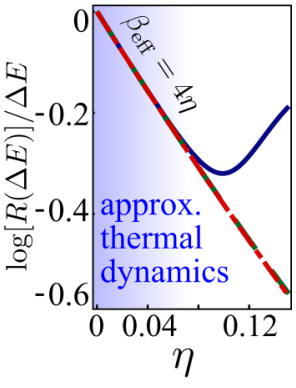

FIG. 4. Asymmetry of the rates. We set $\Omega=0.1, \omega=0.1, \kappa=$ 1. (a) Ratio $R_{i}(\Delta E)=W_{i}(\Delta E) / W_{i}(-\Delta E)$ versus energy difference $|\Delta E|$. For small values of $\eta$ the rate $W_{i}(\Delta E)$ is strongly asymmetric with respect to $\Delta E$, whereas for large $\eta$ we have $W_{i}(\Delta E) \approx$ $W_{i}(-\Delta E)$. (b) The ratio $R_{i}(\Delta E)$ is shown for three different values of $\Delta E=0.02,3,8$ and the values of $g_{k}$ are drawn from a uniform distribution with support in $\left[-g_{0}, g_{0}\right]$ with $g_{0}=6$. At small $\eta$, the ratio $R_{i}(\Delta E)$ approaches one, indicating that configurations are visited with equal probability as for large $\eta$. (c) The three curves in (b) are rescaled according to $\log R_{i}(\Delta E) / \Delta E$. Their asymptotic collapse in the limit $\eta \rightarrow 0$ highlights the existence of a unique inverse temperature $\beta_{\text {eff }}$ which governs the dynamics when $\eta$ is sufficiently small.

for different values of $\Delta E$ and show that different curves collapse to a single (negative) inverse temperature $-\beta_{\text {eff }}$ up to the edge of case (i). At $\eta=0$ we find that $\beta_{\text {eff }}$ approaches zero, leading to an infinite-temperature dynamics. This is reasonable, since in this limit the bosonic gain rate approaches the loss rate. This implies the population of arbitrarily high Fock states, effectively heating the bosons. The latter then act as a high-temperature bath on the spins. If, however, $1 / \beta_{\text {eff }}$ remains small or comparable with the energy gap from the ground states of Eq. (5) - which on average is of order $g^{2}$, meaning $\left.4 \eta g^{2} \geqslant 1\right)$-then an effective low-temperature dynamics is realized.

In case (ii), the ratio $R_{i}(\Delta E) \approx\left(\Delta E-g_{i}^{2}\right)^{4} /\left(\Delta E+g_{i}^{2}\right)^{4}$ tends to increase towards 1 as $\Delta E$ grows. Typically, the available $\Delta E_{i}$ s populate both range (i) and (ii), implying the presence of type (ii) processes which do not follow the same low-temperature rules obeyed by the "small-jump" ones. Provided the number of spins $N$ is not too large, these nonthermal processes constitute, however, a small perturbation for the following two reasons: first, the distribution of energy jumps is peaked around 0 , implying that, if the parameters are adequately chosen, then most jumps lie in regime (i). Second, since the rates are decreasing functions of $\left|\Delta E+g_{i}^{2}\right|$, type (ii) processes occur at smaller rates than the type (i), thermal ones. A numerically exact analysis of the classical master equation for $N=10$, displayed in Figs. 2(b) and 2(c), indeed shows that the effect of the nonthermal processes is sufficiently weak to avoid having a significant population of high-energy states in the long-time limit. The statistical energy distribution tends instead to become concentrated on low-energy configurations, highlighting a clear bias of the dynamics towards cooling, as compared, e.g., to the $\eta \gg 1$ case in Fig. 2(a).

We remark that our considerations above are qualitatively independent of the choice of distribution for the couplings. 


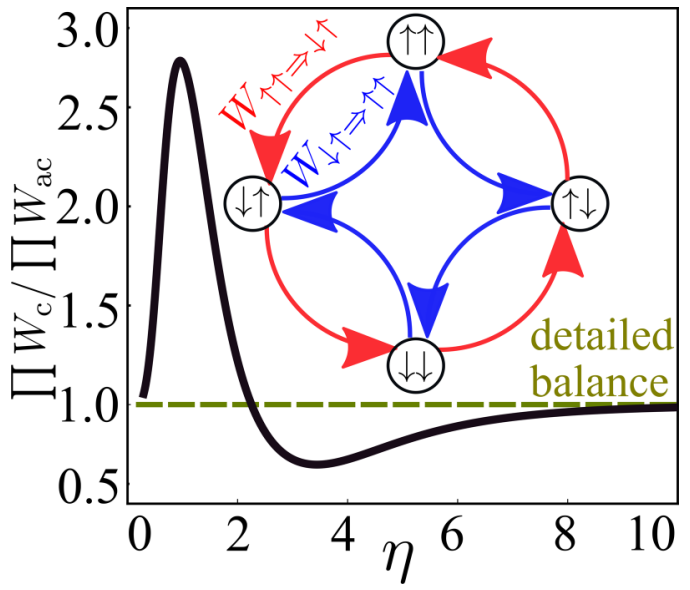

FIG. 5. Nonequilibrium regime. Kolmogorov criterion in a twospin subspace with uniformly distributed couplings; the displayed quantity is the ratio of the product $\prod W_{\mathrm{c}}$ of the four rates encountered when performing the loop clockwise (blue arrows) divided by the analogous counter-clockwise (red arrows) product $\prod W_{\mathrm{ac}}$, plotted as a function of $\eta$. This ratio is 1 (signalling detailed balance conditions) only for large and very small values of $\eta$. The intermediate point $\eta \approx 2$ where $\prod W_{\mathrm{c}}=\prod W_{\mathrm{ac}}$ can be safely ignored for the following reason: for systems with more than two spins there are multiple loops in configuration space and Kolmogorov's criterion is never satisfied simultaneously for all of them (except in the extremal limits $\eta \rightarrow 0$ and $\eta \rightarrow \infty)$ and the dynamics does not obey detailed balance. The parameters are: $\omega=1, \Omega=0.1, \kappa=1$, and $g_{0}=4$.

The latter can influence instead the quantitative aspects, in particular the distribution of the energy jumps, and in turn what portion of them belongs to either the thermal or the nonthermal type. This can shed some light on the difference highlighted in Fig. 3: in fact, it is reasonable to expect, among the two distributions with equal variance, that the uniform one will typically produce smaller (in modulus) couplings than the bimodal one, which is peaked instead away from the origin. Intuitively, the energies Eq. (5) and the energy jumps, quadratic in the couplings, will generically be smaller (in modulus) in the former than in the latter. Given that the parameter $\eta=0.1$, and hence the effective (inverse) temperature $\beta_{\text {eff }}=4 \eta$, is the same for both cases displayed in Fig. 3, it follows that the proportion in which higher-energy states will be populated will typically be greater for uniformly distributed couplings than for the bimodal ones. The population of the ground state reflects this, being smaller in the former case than in the latter.

\section{BREAKDOWN OF DETAILED BALANCE}

Outside the thermal regimes the dynamics is not an equilibrium one, i.e., it does not obey detailed balance. This can be proved via Kolmogorov's criterion [42] which we analyze for the loop formed in the configuration space of a two-spin system (see Fig. 5): $(\uparrow \uparrow) \rightarrow(\uparrow \downarrow) \rightarrow(\downarrow \downarrow) \rightarrow(\downarrow \uparrow) \rightarrow(\uparrow \uparrow)$. To this end we investigate the ratio between the product of the rates for the clockwise (blue arrows) cycle and the corresponding product for the counter-clockwise (red arrows) one. This ratio goes to 1 when $\eta \rightarrow \infty$ and also when $\eta \rightarrow 0$, signaling the emergence of the infinite-temperature dynamics. For different values $\eta$ the ratio is typically different from one, which indicates the persistence of probability currents in the stationary state and the absence of detailed balance.

\section{CONCLUSIONS}

We have studied a disordered dissipative spin-boson system in the limit of strong coupling and weak driving, which can for example be implemented on trapped ion quantum simulators. Many aspects of the emerging physics can be understood in terms of a disordered fully connected Ising model whose state evolves according to a rate equation. In general, the dynamics violates detailed balance, and the system is thus out of equilibrium. However, we could identify parameter regimes in which the evolution is effectively thermal. Among them is one where predominantly low-energy configurations are populated, which mimics the action of a low-temperature dynamics. We have compared two cases in which the couplings have been randomly generated from either a uniform or a bimodal distribution. The latter case typically displays a more effective ability to cool the spins, typically achieving more than $50 \%$ population of the ground state in the stationary limit. In the future it would be interesting to see whether this effective cooling mechanism permits to access low-energy states or even ground states of complex spin networks. This might open an elegant way for encoding and solving computationally hard problems [18] or associative memories [19] through Ising energy functions and an appropriate (thermal) dynamics on quantum simulators.

\section{ACKNOWLEDGMENTS}

The research leading to these results has received funding from the European Research Council under the European Unions Seventh Framework Programme (FP/20072013)/ERC Grant Agreement No. 335266 (ESCQUMA), the EPSRC Grant No.EP/R04340X/1 via the QuantERA project "ERyQSenS," and from the University of Nottingham through a Nottingham Research Fellowship (M.M.). P.R. acknowledges funding by the European Union through the H2020-MCIF Grant No. 766442. I.L. gratefully acknowledges funding through the Royal Society Wolfson Research Merit Award.

\section{APPENDIX A: DERIVATION OF THE RATES}

In this section we provide details on the derivation of Eq. (3). We first consider the evolution of the state $\rho(t)$ as $\dot{\rho}=\mathcal{L}_{0}(\rho)+\mathcal{L}_{1}(\rho)$, with $\mathcal{L}_{0}=\mathcal{L}-\mathcal{L}_{1}$ and $\mathcal{L}_{1}(\cdot)=$ $-i \Omega\left[\sum_{i} \hat{\sigma}_{i}^{x}, \cdot\right]$. Second, we assume the stationary state of $\mathcal{L}_{0}$ of the form $\rho_{\text {stat }}=\sum_{\vec{\sigma}} p_{\vec{\sigma}} \rho_{\vec{\sigma}}|\vec{\sigma}\rangle\langle\vec{\sigma}|$, where $|\vec{\sigma}\rangle=$ $\left\{\sigma_{1}, \ldots, \sigma_{N}\right\}$, with $\sigma_{i}= \pm 1$ and $\hat{\sigma}_{i}^{z}|\vec{\sigma}\rangle=\sigma_{i}|\vec{\sigma}\rangle, p_{\vec{\sigma}}$ are a set of classical probabilities and $\rho_{\sigma}$ is the corresponding bosonic state, that we assume to be a gaussian state. Considering $\mathcal{L}_{1}$ perturbatively with respect to $\mathcal{L}_{0}$, and projecting the dynamics onto the stationary manifold of $\mathcal{L}_{0}$, we exploit the Nakajima- 
Zwanzig formalism to write the evolution of the spin as

$$
\begin{aligned}
P \dot{\rho}^{\text {spin }}(t)= & \operatorname{Tr}_{B} \int_{0}^{+\infty} d t^{\prime} P \mathcal{L}_{1} e^{\mathcal{L}_{0} T^{\prime}} \mathcal{L}_{1} \rho_{\text {stat }}(t) \\
= & \Omega^{2} \sum_{\{\vec{\sigma}\}} p_{\vec{\sigma}}(t) \sum_{i} \int_{0}^{+\infty} d t^{\prime} \sum_{j= \pm} \operatorname{Tr}_{B}\left[e^{\mathcal{V}_{\vec{\sigma}, i}^{j} t^{\prime}}\left(\rho_{\vec{\sigma}}\right)\right] \\
& \times\left(\hat{\sigma}_{i}^{x}|\vec{\sigma}\rangle\left\langle\vec{\sigma}\left|\hat{\sigma}_{i}^{x}-\right| \vec{\sigma}\right\rangle\langle\vec{\sigma}|\right),
\end{aligned}
$$

where $P \dot{\rho}^{\text {spin }}(t)=\operatorname{Tr}_{B}\left[\dot{\rho}_{\text {stat }}(t)\right]=\sum_{\vec{\sigma}} \dot{p}_{\vec{\sigma}}(t)|\vec{\sigma}\rangle\langle\vec{\sigma}|, \operatorname{Tr}_{B}$ is the partial trace over the boson, and we have defined the spin-configuration-dependent superoperators

$$
\begin{aligned}
\mathcal{V}_{\vec{\sigma}, i}^{ \pm}(\cdot)= & -i \omega\left[\hat{a}^{\dagger} \hat{a}, \cdot\right]+\mathcal{D}_{\gamma}(\cdot)+\mathcal{D}_{\kappa}(\cdot) \\
& -i g_{i} \mathcal{M}_{i}\left[\left(\hat{a}^{\dagger}+\hat{a}\right), \cdot\right] \pm i g_{i} \sigma_{i}\left\{\left(\hat{a}^{\dagger}+\hat{a}\right), \cdot\right\},
\end{aligned}
$$

with $\mathcal{M}_{i}=\sum_{l \neq i} g_{l} \sigma_{l}$, and $\mathcal{D}_{\gamma}, \mathcal{D}_{\kappa}$ the dissipative terms representing cooling and heating, respectively. By projecting Eq. (A1) on a state $\left|\vec{\sigma}^{\prime}\right\rangle$, the dynamics reduces to the evolution of the classical probabilities ruled by a master equation whose general form is the following:

$$
\dot{p}_{\vec{\sigma}}=\sum_{\vec{\sigma}^{\prime}}\left(W_{\vec{\sigma}^{\prime} \rightarrow \vec{\sigma}} p_{\vec{\sigma}^{\prime}}-W_{\vec{\sigma} \rightarrow \vec{\sigma}^{\prime}} p_{\vec{\sigma}}\right),
$$

where $W_{\vec{\sigma} \rightarrow \vec{\sigma}^{\prime}}=\Omega^{2} \int_{0}^{+\infty} d t \sum_{j= \pm} \operatorname{Tr}_{B}\left[e^{\mathcal{V}_{\vec{\sigma}, i}^{j} t}\left(\rho_{\vec{\sigma}}\right)\right]$, is the transition rate for the switching $\vec{\sigma} \rightarrow \vec{\sigma}^{\prime}$ and it allows only single spin-flip processes.

We can now go ahead in evaluating explicitly the expression for the rates. Exploiting the superoperator's properties, we can substitute inside the trace $e^{\mathcal{V}_{\vec{\sigma}, i}^{ \pm}}\left(\rho_{\vec{\sigma}}\right) \rightarrow\left(e^{\mathcal{V}_{\vec{\sigma}, i}^{ \pm, *}} \mathbb{1}\right) \rho_{\vec{\sigma}}$, with $\mathcal{V}_{\vec{\sigma}, i}^{ \pm, *}$ the adjoint superoperator of $\mathcal{V}_{\vec{\sigma}, i}^{ \pm}$and $\mathbb{1}$ the identity operator. It is worth noticing that the identity operator can be expressed in terms of a generalised displacement operator of field coherent states as $\mathbb{1}=\hat{D}(0)$, where $\hat{D}(\tau)=$ $e^{\alpha(\tau) \hat{a}^{\dagger}-\beta(\tau)^{*} \hat{a}} e^{-\gamma(\tau)}$ with $\alpha(0)=\beta(0)=\gamma(0)=0$. We then verify that the displacement operator $\hat{D}(0)$ is mapped into the generalised one $\hat{D}(\tau)$ by applying the adjoint superoperator

$$
2\left(\begin{array}{c}
-\left(\left\langle\hat{a}^{2}\right\rangle_{G}-\langle\hat{a}\rangle_{G}^{2}\right) \\
\frac{1}{2}\left(\left\langle\hat{a}^{\dagger} \hat{a}\right\rangle_{c}+\left\langle\hat{a} \hat{a}^{\dagger}\right\rangle_{G}\right)-\left\langle\hat{a}^{\dagger}\right\rangle_{G}\langle\hat{a}\rangle_{G}
\end{array}\right.
$$

By applying the definition Eq. (A5), with expectation values of the operators obtained considering the Lindblad operator $\mathcal{L}_{0}$, we get

$$
\begin{aligned}
\chi_{\vec{\sigma}, i}^{ \pm}(\tau)= & \exp \left\{-\frac{1+\theta}{2(1-\theta)}\left|\alpha_{\sigma_{i}}^{ \pm}(\tau)\right|^{2}\right. \\
& \left.+\frac{2 i g_{i} \mathcal{M}}{\omega}\left(\frac{\alpha_{\sigma_{i}}^{ \pm}(\tau)}{\eta-2 i}+\frac{\alpha_{\sigma_{i}}^{ \pm *}(\tau)}{\eta+2 i}\right)\right\},
\end{aligned}
$$

where $\mathcal{M}=\sum_{l} g_{l} \sigma_{l}$. Thus, the expression of the rate reads

$$
\begin{aligned}
W_{\vec{\sigma} \rightarrow \vec{\sigma}^{\prime}} & =\frac{\Omega^{2}}{\omega} \int_{0}^{+\infty} d \tau \sum_{j= \pm} e^{-\gamma_{\sigma_{i}}^{j}(\tau)} \chi_{\vec{\sigma}, i}^{j}(\tau) \\
& =\frac{2 \Omega^{2}}{\omega} \int_{0}^{\infty} d \tau e^{-\frac{2 g_{i}^{2} v}{\omega^{2}}[f(\tau)+\tau]} \cos \left[16 \frac{\Delta E_{i} \tau-g_{i}^{2} s(\tau)}{\omega^{2}\left(\eta^{2}+4\right)}\right],
\end{aligned}
$$

$\mathcal{V}_{\vec{\sigma}, i}^{ \pm}$indeed, by considering the differential equation

$$
\frac{d}{d \tau}\left[\hat{D}_{\vec{\sigma}, i}^{ \pm}(\tau)\right]=\mathcal{V}_{\vec{\sigma}, i}^{ \pm *}\left[\hat{D}_{\vec{\sigma}, i}^{ \pm}(\tau)\right],
$$

we obtain the solutions for the functions $\alpha_{\sigma_{i}}^{+}(\tau)=\alpha_{-\sigma_{i}}^{-}(\tau)$, $\beta_{\sigma_{i}}^{+}(\tau)=\beta_{-\sigma_{i}}^{-}(\tau), \gamma_{\sigma_{i}}^{+}(\tau)=\gamma_{-\sigma_{i}}^{-}(\tau)$. For initial conditions $\alpha_{\sigma_{i}}^{ \pm}(0)=\beta_{\sigma_{i}}^{ \pm}(0)=\gamma_{\sigma_{i}}^{ \pm}(0)=0$, we get

$$
\begin{aligned}
\alpha_{\sigma_{i}}^{+}(\tau) & =\left[\beta_{\sigma_{i}}^{+}(t)\right]^{*}=\frac{i 4 g_{i} \sigma_{i}}{\omega(\eta-2 i)}\left[1-e^{\left(i-\frac{\eta}{2}\right) \tau}\right], \\
\gamma_{\sigma_{i}}^{+}(\tau) & =\frac{2 g_{i}^{2} v}{\omega^{2} \eta}\left[f_{1}(\tau)+\tau\right]+\frac{i g_{i} \sigma_{i} \mathcal{M}_{i}}{\omega^{2}\left(\eta^{2}+4\right)}[s(\tau)+\tau], \\
f_{1}(\tau) & =\frac{1-e^{\eta \tau}}{\eta}-\frac{4 \eta\left[1-e^{-\frac{\eta}{2} \tau} \cos (\tau)\right]-8 e^{-\frac{\eta}{2} \tau} \sin (\tau)}{\eta^{2}+4}, \\
s(\tau) & =\frac{4 \eta\left[e^{-\frac{\eta}{2} \tau} \cos (\tau)-1\right]+\left(\eta^{2}-4\right) e^{-\frac{\eta}{2} \tau} \sin (\tau)}{\eta^{2}+4},
\end{aligned}
$$

where we have defined the dimensionless time $\tau=t \omega$, and $\eta=(\gamma-\kappa) / \omega, \theta=\kappa / \gamma \in[0,1)$, and

$$
v=\frac{4(1+\theta) \eta}{\left(\eta^{2}+4\right)(1-\theta)}=\frac{4\left(2 \frac{\kappa}{\omega}+\eta\right)}{\eta^{2}+4} .
$$

The previous steps allow us to write the partial trace over the boson as $\operatorname{Tr}_{B}\left[e^{\mathcal{V}_{\vec{\sigma}, i}^{ \pm}}\left(\rho_{\vec{\sigma}}\right)\right]=e^{-\gamma_{\sigma_{i}}^{ \pm}(\tau)} \operatorname{Tr}_{B}\left[\hat{D}_{\vec{\sigma}, i}^{ \pm}(\tau) \rho_{\vec{\sigma}}\right]$. We recall that the bosonic state $\rho_{\vec{\sigma}}$ has been assumed to be a gaussian state. In this case, we recognize the quantity $\operatorname{Tr}_{B}\left[\hat{D}_{\vec{\sigma}, i}^{ \pm}(\tau) \rho_{\vec{\sigma}}\right]$ as the characteristic function $\chi_{\vec{\sigma}, i}^{ \pm}(\tau)$ of the state $\rho_{\vec{\sigma}}$. The expression of the characteristic function for a generic Gaussian state $\rho_{G}$ reads

$$
\chi_{\rho_{G}}[\alpha(\tau)]=e^{-\frac{1}{4} \vec{\alpha}^{T}(\tau) \Sigma \vec{\alpha}(\tau)+\alpha(\tau)\left\langle\hat{a}^{\dagger}\right\rangle_{G}-\alpha^{*}(\tau)\langle\hat{a}\rangle_{G}},
$$

where $\vec{\alpha}^{T}=\left(\alpha(\tau), \alpha^{*}(\tau)\right),\langle\cdot\rangle_{G}$ is the expectation value performed over the state $\rho_{G}$, and $\Sigma$ represents the covariance matrix, which reads

$$
\left.\begin{array}{c}
\frac{1}{2}\left(\left\langle\hat{a}^{\dagger} \hat{a}\right\rangle_{G}+\left\langle\hat{a} \hat{a}^{\dagger}\right\rangle_{G}\right)-\left\langle\hat{a}^{\dagger}\right\rangle_{G}\langle\hat{a}\rangle_{G} \\
-\left(\left\langle\hat{a}^{\dagger}{ }^{2}\right\rangle_{G}-\left\langle\hat{a}^{\dagger}\right\rangle_{G}^{2}\right)
\end{array}\right) .
$$

$$
f(\tau)=\frac{-2 \eta^{2}+8}{\eta\left(\eta^{2}+4\right)}\left[1-e^{-\frac{\eta}{2} \tau} \cos (\tau)\right]-\frac{8 e^{-\frac{\eta}{2} \tau}}{\eta^{2}+4} \sin (\tau),
$$

where $\Delta E_{i}=g_{i} \sigma_{i} \sum_{l \neq i} g_{l} \sigma_{l}$ retains the dependence on the spin configuration.

\section{APPENDIX B: APPROXIMATE EXPRESSION OF THE RATE FOR $\eta$ SMALL}

For sufficiently small $\eta \lesssim 1$, the exponent appearing in $W_{\vec{\sigma} \rightarrow \vec{\sigma}^{\prime}}$ is dominated by

$$
f(\tau) \approx \frac{2}{\eta}[1-\cos (\tau)],
$$


implying that the integrand is quickly suppressed as $\tau$ grows. We therefore perform an expansion around $\tau=0$, which yields

$$
\tau+f(\tau)=\left(\eta^{2}+4\right)\left(\frac{1}{4 \eta} \tau^{2}-\frac{1}{24} \tau^{3}-\frac{4-\eta^{2}}{192 \eta} \tau^{4}\right)+O\left(\tau^{5}\right)
$$

and

$$
s(\tau)=-\tau+\frac{\eta^{2}+4}{24} \tau^{3}-\eta \frac{\eta^{2}+4}{96} \tau^{4}+O\left(\tau^{5}\right) .
$$

From the first term in Eq. (B2) we see that the integrand is strongly suppressed on scales $\tau \gtrsim \sqrt{\eta}$. Noticing that in the Taylor expansion of $\tau+f(\tau)$ odd coefficients are finite, whereas even ones are $O(1 / \eta)$ for $\eta \rightarrow 0$ and introducing the rescaled integration variable $z=\tau \sqrt{\eta}$ we see that higher orders are perturbations of order $O\left(\eta^{n+1 / 2} z^{2 n+1}, \eta^{n-1} z^{2 n}\right)$ and can be neglected. Similar considerations can be applied to $s(\tau)$, which can be therefore also approximated with its leading order $-\tau$. In the following, for simplicity we set $\omega=1$, remembering that our "energy" $\Delta E_{i}$ is actually measured by construction in units of $\omega^{2}$. Additionally, we introduce the shorthand

$$
\Gamma_{i}=\frac{16}{\eta^{2}+4}\left(\Delta E_{i}+g_{i}^{2}\right),
$$

so that, by keeping only the lowest orders of the expansion in $\tau$, we can approximate our rate as

$$
W_{\vec{\sigma} \rightarrow \vec{\sigma}^{\prime}} \approx 2 \Omega^{2} \int_{0}^{\infty} d \tau e^{-2 \frac{g_{i}^{2}(2 \kappa+\eta)}{\eta} \tau^{2}} \cos \left(\Gamma_{i} \tau\right),
$$

which can be integrated to give the closed expression

$$
W_{\vec{\sigma} \rightarrow \vec{\sigma}^{\prime}} \approx \Omega^{2} \sqrt{\frac{\pi \eta}{2 g_{i}^{2}(2 \kappa+\eta)}} e^{-\frac{\eta \Gamma_{i}^{2}}{8 g_{i}^{2}(2 \kappa+\eta)}} .
$$

From the expression above one can retrieve Eq. (7) by taking the leading behavior in the limit $\eta \rightarrow 0$. It is worth remarking that the exponent can be rewritten as

$$
\begin{aligned}
-\frac{\eta \Gamma_{i}^{2}}{8 g_{i}^{2}(2 \kappa+\eta)} & =\frac{128 \eta}{(2 \kappa+\eta)\left(\eta^{2}+4\right)^{2}}\left[-\frac{\left(\Delta E_{i}+g_{i}^{2}\right)^{2}}{4 g_{i}^{2}}\right] \\
& =\frac{128 \eta}{(2 \kappa+\eta)\left(\eta^{2}+4\right)^{2}}\left[E(\vec{\sigma})-\frac{\sum_{j} g_{j}^{2}}{4}\right] \\
& =\beta_{\text {eff }} E(\vec{\sigma})-\text { const. }
\end{aligned}
$$

highlighting the "thermal" structure of the rates. Note that, to obtain the approximation Eq. (B5), we have assumed that we can resum the Taylor expansion of the original cosine to the function $\cos \left(\Gamma_{i} \tau\right)$, whose series only coincides with the former up to $O\left(\tau^{2}\right)$. This is only valid as long as the cosine does not oscillate significantly before the other Gaussian term suppresses the integrand; in other words, Eq. (B6) should be valid up to values of $\Gamma_{i}$ of the order of $\sim 1 / \sqrt{\eta}$. Since we wish to understand the behavior of the rates as functions of the energy difference $\Delta E_{i}$ without restrictions imposed by the other parameters (like $\eta$ ), we need to account for energies which exceed this range. To do this, we extract the asymptotic behavior of the rate for $\Gamma_{i} \rightarrow \infty$. We start by rewriting the integrand in $W$ as

$$
\begin{aligned}
I\left(\Gamma_{i}, \tau\right) & \equiv \operatorname{Re}\left\{e^{-2 \frac{g_{i}^{2} \nu}{\omega^{2}}(f(\tau)+\tau)} e^{i \Gamma_{i} \tau-16 i \frac{g_{i}^{2}}{\eta^{2}+4}(s(\tau)+\tau)}\right\} \\
& =\operatorname{Re}\left\{A(\tau) e^{i \Gamma_{i} \tau}\right\} .
\end{aligned}
$$

We now use the result that, if the function $A$ admits a small $\tau$ expansion

$$
A(\tau)=\sum_{n=0}^{\infty} a_{n} \tau^{n}
$$

then asymptotically in the limit $\Gamma_{i} \rightarrow \infty$ one finds

$$
\int_{0}^{\infty} d \tau A(\tau) e^{i \Gamma_{i} \tau}=\sum_{n=0}^{\infty} i^{n} n ! a_{n} \Gamma_{i}^{-n-1} .
$$

Referring back to Eq. (B8), the leading term in this expansion corresponds to the lowest $n$ for which one finds a nonvanishing real part. In particular, we note that $\operatorname{Re}\left[a_{n} i^{n+1}\right]$ equals $(-1)^{l+1} \operatorname{Re}\left[a_{2 l+1}\right]$ if $n=2 l+1$ is odd, and $(-1)^{l+1} \operatorname{Im}\left[a_{2 l}\right]$ if $n=2 l$ is even. For our function we find

$$
\begin{aligned}
& a_{0}=1, \\
& a_{1}=0, \\
& a_{2}=-2 g_{i}^{2} \frac{2 \kappa+\eta}{\eta}, \\
& a_{3}=\frac{g_{i}^{2}}{3}[2 \kappa+\eta-2 i] .
\end{aligned}
$$

The leading behavior in the large $\Gamma_{i}$ limit is therefore determined by $\operatorname{Re}\left[a_{3}\right]$, implying

$$
\begin{aligned}
W_{\vec{\sigma} \rightarrow \vec{\sigma}^{\prime}} & =2 \Omega^{2} \int_{0}^{\infty} d \tau I\left(\Gamma_{i}, \tau\right) \\
& \approx 4 \Omega^{2} g_{i}^{2}(2 \kappa+\eta)\left[\frac{\eta^{2}+4}{16\left(\Delta E_{i}+g_{i}^{2}\right)}\right]^{4} .
\end{aligned}
$$

To provide a very crude estimate of where the change from the two regimes characterized by Eqs. (B6) ("small $\Gamma_{i}$ ") and (B13) ("large $\Gamma_{i}$ ") occurs, we evaluate the point where the two asymptotic expressions cross (for $\eta$ sufficiently small): Setting

$$
4 g_{i}^{2}(2 \kappa+\eta) \Gamma_{i}^{-4}=\sqrt{\frac{\pi \eta}{2 g_{i}^{2}(2 \kappa+\eta)}} e^{-\frac{\eta \Gamma_{i}^{2}}{8 g_{i}^{2}(2 \kappa+\eta)}},
$$

we find

$$
\Gamma_{i}^{2} \approx \frac{4 g_{i}^{2}(2 \kappa+\eta)}{\eta}\left[\log A+4 \log \left(\frac{1}{2} \log A\right)\right],
$$

where $A=32 g_{i}^{2} \pi(2 \kappa+\eta) \eta^{-3}$.
[1] A. Blais, R.-S. Huang, A. Wallraff, S. M. Girvin, and R. J. Schoelkopf, Phys. Rev. A 69, 062320 (2004).
[2] H. Zheng, D. J. Gauthier, and H. U. Baranger, Phys. Rev. Lett. 111, 090502 (2013) 
[3] J. I. Cirac and P. Zoller, Phys. Rev. Lett. 74, 4091 (1995).

[4] D. Leibfried, R. Blatt, C. Monroe, and D. Wineland, Rev. Mod. Phys. 75, 281 (2003).

[5] A. F. Kockum, A. Miranowicz, S. De Liberato, S. Savasta, and F. Nori, Nature Rev. Phys. 1, 19 (2019).

[6] E. Farhi, J. Goldstone, S. Gutmann, J. Lapan, A. Lundgren, and D. Preda, Science 292, 472 (2001).

[7] V. S. Denchev, S. Boixo, S. V. Isakov, N. Ding, R. Babbush, V. Smelyanskiy, J. Martinis, and H. Neven, Phys. Rev. X 6, 031015 (2016).

[8] P. Strack and S. Sachdev, Phys. Rev. Lett. 107, 277202 (2011).

[9] S. Gopalakrishnan, B. L. Lev, and P. M. Goldbart, Phys. Rev. Lett. 107, 277201 (2011).

[10] P. Rotondo, E. Tesio, and S. Caracciolo, Phys. Rev. B 91, 014415 (2015).

[11] P. Rotondo, M. Cosentino Lagomarsino, and G. Viola, Phys. Rev. Lett. 114, 143601 (2015).

[12] Emanuele G. Dalla Torre, S. Diehl, M. D. Lukin, S. Sachdev, and P. Strack, Phys. Rev. A 87, 023831 (2013).

[13] E. Fiorelli, P. Rotondo, M. Marcuzzi, J. P. Garrahan, and I. Lesanovsky, Phys. Rev. A 99, 032126 (2019).

[14] P. Rotondo, M. Marcuzzi, J. P. Garrahan, I. Lesanovsky, and M. Müller, J. Phys. A: Math. Theor. 51, 115301 (2018).

[15] M. Buchhold, P. Strack, S. Sachdev, and S. Diehl, Phys. Rev. A 87, 063622 (2013).

[16] V. D. Vaidya, Y. Guo, R. M. Kroeze, K. E. Ballantine, A. J. Kollár, J. Keeling, and B. L. Lev, Phys. Rev. X 8, 011002 (2018).

[17] Y. Guo, R. M. Kroeze, V. V. Vaidya, J. Keeling, and B. L. Lev, Phys. Rev. Lett. 122, 193601 (2019).

[18] F. Barahona, J. Phys. A: Math. Gen. 15, 3241 (1982).

[19] J. J. Hopfield, Proc. Natl. Acad. Sci. USA 79, 2554 (1982).

[20] B. M. Garraway, Philos. Trans. R. Soc. London A 369, 1137 (2011).

[21] P. Kirton, M. M. Roses, J. Keeling, and E. G. Dalla Torre, Adv. Quant. Technol. 2, 1800043 (2019).

[22] K. Hepp and E. H. Lieb, Ann. Phys. 76, 360 (1973).
[23] K. Hepp and E. H. Lieb, Phys. Rev. A 8, 2517 (1973).

[24] E. G. Dalla Torre, Y. Shchadilova, E. Y. Wilner, M. D. Lukin, and E. Demler, Phys. Rev. A 94, 061802(R) (2016).

[25] P. Kirton and J. Keeling, Phys. Rev. Lett. 118, 123602 (2017).

[26] Z. Zhang, C. H. Lee, R. Kumar, K. J. Arnold, S. J. Masson, A. L. Grimsmo, A. S. Parkins, and M. D. Barrett, Phys. Rev. A 97, 043858 (2018).

[27] G. D. Chiara, G. Landi, A. Hewgill, B. Reid, A. Ferraro, A. J. Roncaglia, and M. Antezza, New J. Phys. 20, 113024 (2018).

[28] A. Levy and R. Kosloff, Europhys. Lett. 107, 20004 (2014).

[29] D. Porras and J. I. Cirac, Phys. Rev. Lett. 92, 207901 (2004).

[30] I. Aedo and L. Lamata, Phys. Rev. A 97, 042317 (2018).

[31] D. Lv, S. An, Z. Liu, J.-N. Zhang, J. S. Pedernales, L. Lamata, E. Solano, and K. Kim, Phys. Rev. X 8, 021027 (2018).

[32] A. Safavi-Naini, R. J. Lewis-Swan, J. G. Bohnet, M. Gärttner, K. A. Gilmore, J. E. Jordan, J. Cohn, J. K. Freericks, A. M. Rey, and J. J. Bollinger, Phys. Rev. Lett. 121, 040503 (2018).

[33] A. Mezzacapo, U. Las Heras, J. Pedernales, L. DiCarlo, E. Solano, and L. Lamata, Sci. Rep. 4, 7482 (2014).

[34] G.-D. Lin, S.-L. Zhu, R. Islam, K. Kim, M.-S. Chang, S. Korenblit, C. Monroe, and L.-M. Duan, Europhys. Lett. 86, 60004 (2009).

[35] V. V. Albert and L. Jiang, Phys. Rev. A 89, 022118 (2014).

[36] P. Degenfeld-Schonburg and M. J. Hartmann, Phys. Rev. B 89, 245108 (2014).

[37] M. Marcuzzi, J. Schick, B. Olmos, and I. Lesanovsky, J. Phys. A: Math. Theor. 47, 482001 (2014).

[38] H. P. Breuer and F. Petruccione, The Theory of Open Quantum Systems (Oxford University Press, Great Clarendon Street, 2002).

[39] B. Everest, M. Marcuzzi, J. P. Garrahan, and I. Lesanovsky, Phys. Rev. E 94, 052108 (2016).

[40] Note, that in this notation if $W_{\vec{\sigma} \rightarrow \vec{\sigma}^{\prime}} \equiv W_{i}(\Delta E)$, then $W_{\vec{\sigma}^{\prime} \rightarrow \vec{\sigma}} \equiv$ $W_{i}(-\Delta E)$

[41] H. Dai and D. Naylor, Proc. R. Soc. London A 436, 109 (1992).

[42] R. K. P. Zia and B. Schmittmann, J. Stat. Mech. (2007) P07012. 